Antibiotikatherapie der Tuberkulose

\title{
Einige Patienten profitieren von Vitamin $D_{3}$
}

Martineau AR et al. High-dose vitamin D3 during intensive-phase antimicrobial treatment of pulmonary tuberculosis: a double-blind randomised controlled trial. Lancet 2011;377:242-250

\section{Hintergrund}

Calcitriol, der aktive Metabolit von Vitamin $\mathrm{D}$, induziert in vitro eine antimykobakterielle Aktivität [1]. In einigen Fallserien wurde berichtet, dass Vitamin D in Dosen zwischen 0,625 und 2,5 $\mathrm{mg} / \mathrm{d}$ das Ansprechen auf die antimikrobielle Behandlung bei Patienten mit Lungentuberkulose verbessert hat. Randomisierte kontrollierte Studien mit bis zu 0,125 $\mathrm{mg} / \mathrm{d}$ Vitamin D oder Äquivalent bei aktiver Tuberkulose hatten keinen klinischen Nutzen ergeben [2]. Jedoch wurde in einer indonesischen Studie mit einer höheren Vitamin-D-Dosis $(0,25 \mathrm{mg} / \mathrm{d})$ eine verbesserte Rate der Konversion des Sputumausstrichs innerhalb von sechs Wochen gefunden [3]. Die Konversion des mikroskopisch untersuchten Sputumausstrichs ist allerdings als Biomarker für den Behandlungserfolg bei Lungentuberkulose weniger sensitiv und spezifisch als eine Kultur.

\section{Methodik}

In eine von der British Lung Foundation unterstützten und in zehn Londoner Zentren durchgeführten, randomisierten,

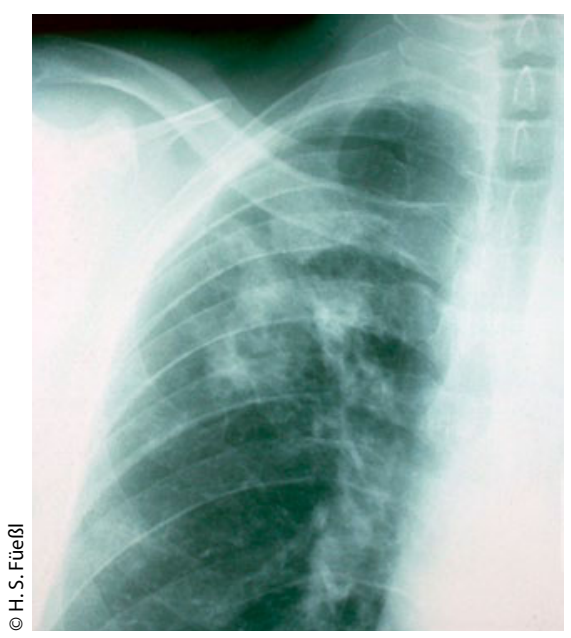

Kavernöse Lungentuberkulose. kontrollierten Doppelblindstudie wurden 146 Patienten mit frisch diagnostizierter Lungentuberkulose und mikroskopischem Nachweis säurefester Stäbchen im Sputumausstrich eingeschlossen. Sie erhielten zusätzlich zu ihrer StandardAntibiotikabehandlung am Beginn sowie an den Tagen 14, 28 und 42 entweder 2,5 mg Vitamin $\mathrm{D}_{3}$ (Cholecalciferol) oder Placebo.

Der primäre Endpunkt war die Zeit vom Beginn der antibiotischen Behandlung bis zur Konversion der Sputumkultur. Hyperkalzämie (korrigiertes SerumKalzium > 2,65 mmol/l) war der primäre Sicherheitsendpunkt.

Die Patienten wurden hinsichtlich Polymorphismen der TaqI- und FokI-Gene im Vitamin-D-Rezeptor genotypisiert, um Interaktionen zwischen dem Vitamin-DRezeptor-Genotyp und dem Ansprechen auf Vitamin $D_{3}$ zu analysieren.

\section{Ergebnisse}

In die primäre Wirksamkeitsanalyse wurden 126 Patienten einbezogen. Der Median der Zeit bis zur Konversion der Sputumkultur betrug 36,0 Tage in der Interventions- und 43,5 Tage in der Placebogruppe (adjustierte Hazard Ratio [HR]= 1,39; 95\%-Konfidenzintervall [KI]: 0,902,16; $\mathrm{p}=0,14)$.

Der TaqI-Genotyp modifizierte die Wirkung der Vitamin-D-Supplementierung auf die Zeit bis zur Konversion der Sputumkultur $\left(p_{\text {interaction }}=0,03\right.$ ). Eine Verstärkung des Ansprechens wurde nur bei Patienten mit dem tt-Genotyp des TaqIVitamin-D-Rezeptor-Polymorphismus beobachtet (HR = 8,09; 95\%-KI: 1,36$48,01 ; \mathrm{p}=0,02)$. Der FokI-Genotyp modifizierte die Wirkung der Vitamin-DSupplementierung nicht $\left(p_{\text {interaction }}=0,85\right)$.

Eine milde Hyperkalzämie wurde bei bei zwei Patienten der Interventionsgruppe beobachtet, war aber innerhalb von 14
Tagen nach Beendigung der Vitamin- $\mathrm{D}_{3}$ Gabe wieder verschwunden.

Die mittlere 25-Hydroxy-Vitamin-DSerum-Konzentration nach 56 Tagen betrug 101,4 nmol/l in der Interventionsbzw. 22,8 nmol/l in der Placebogruppe (95\%-KI für den Unterschied: 68,6-88,2; $\mathrm{p}<0,0001)$.

\section{Schlussfolgerung}

Die Gabe von vier Dosen Vitamin D zu je 2,5 mg während der Intensivphase der antibiotischen Behandlung einer Lungentuberkulose führte bei der gesamten Studiengruppe nicht zu einer signifikanten Änderung der Zeit bis zur Konversion der Sputumkultur. Bei einer Subgruppe, den Trägern des tt-Genotyps des TaqI-Vitamin-D-Rezeptor-Polymorphismus, wurde jedoch die Konversion der Sputumkultur signifikant beschleunigt. Welcher Mechanismus dieser Gen-Umwelt-Interaktion zugrunde liegt, wird zu klären sein.

\section{Kommentar}

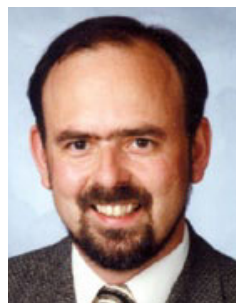

Prof. Dr. med.

Klaus Mörike, Tübingen

Eine Untergruppe von Patienten mit Lungentuberkulose kann von einer Vitamin-D-Supplementierung profitieren. Diese Untergruppe, die Träger des tt-Genotyps des TaqI-Vitamin-D-Rezeptor-Polymorphismus, umfasste ca. $10 \%$ der Studienpopulation. Eine Schlussfolgerung für die anderen Subgruppen ist derzeit noch nicht möglich.

Eine wichtige Frage betrifft die Wirksamkeit von Vitamin D in der Prävention der Aktivierung einer latenten Tuberkulose. Wie der begleitende Kommentar erläutert, stößt eine Präventionsstudie auf größere Hürden als eine Therapiestudie [4].

\section{Literatur}

1. Rook GA et al. Immunology 1986;57:159-163

2. Martineau AR et al. J Steroid Biochem Mol Biol 2007;103:793-798

3. Nursyam EW et al. Acta Med Indones. 2006;38: 3-5

4. Vieth R. Lancet 2011;377:189-190 